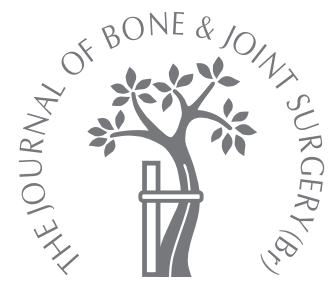

\title{
Enhancement of meniscal repair in the avascular zone using mesenchymal stem cells in a porcine model
}

\author{
A. Q. Dutton, \\ P. F. Choong, \\ J. C-H. Goh, \\ E. H. Lee, \\ J. H. P. Hui
}

From the National

University Health

System, Singapore

\footnotetext{
A. Q. Dutton, FRCS(Orth), Consultant Orthopaedic Surgeon

Department of Orthopaedic Surgery

National Univerrsity Hospital, 5 Lower Kent Ridge Road, Singapore 119074.

P. F. Choong, BSc, MSc, Research Scientist J. C-H. Goh, PhD, Research Professor

E. H. Lee, FRCS(C), Professor J. H. P. Hui, MD, FRCS(Ed), Associate Professor Department of Orthopaedic Surgery

Yong Loo Lin School of Medicine, National University of Singapore, 10 Medical Drive, Singapore 117597.
}

Correspondence should be sent to Professor J. H. P. Hui; e-mail: doshuij@nus.edu.sg

(c)2010 British Editorial Society of Bone and Joint Surgery doi:10.1302/0301-620X.92B1. $22629 \$ 2.00$

$J$ Bone Joint Surg [Br] 2010;92-B:169-75. Received 23 March 2009; Accepted after revision 11 August 2009

We hypothesised that meniscal tears treated with mesenchymal stem cells (MSCs) together with a conventional suturing technique would show improved healing compared with those treated by a conventional suturing technique alone. In a controlled laboratory study 28 adult pigs (56 knees) underwent meniscal procedures after the creation of a radial incision to represent a tear. Group 1 ( $n=9$ ) had a radial meniscal tear which was left untreated. In group 2 ( $n=19$ ) the incision was repaired with sutures and fibrin glue and in group 3 , the experimental group $(n=28)$, treatment was by MSCs, suturing and fibrin glue.

At eight weeks, macroscopic examination of group 1 showed no healing in any specimens. In group 2 no healing was found in 12 specimens and incomplete healing in seven. The experimental group 3 had 21 specimens with complete healing, five with incomplete healing and two with no healing. Between the experimental group and each of the control groups this difference was significant $(p<0.001)$.

The histological and macroscopic findings showed that the repair of meniscal tears in the avascular zone was significantly improved with MSCs, but that the mechanical properties of the healed menisci remained reduced.

Meniscal injuries are common and most procedures for repair give mixed results. With the advent of arthroscopic surgery, partial meniscectomy, in which only the torn portion is removed, has been commonly undertaken. ${ }^{1}$ Removal of the medial meniscus results in a reduction of $50 \%$ to $70 \%$ of the contact area of the femoral condyle and an increase of $100 \%$ in contact stress, ${ }^{2,3}$ with the possibility of degenerative joint disease developing as a result. ${ }^{4,5}$

In an attempt to prevent osteoarthritis, meniscal tears in the peripheral third of the meniscus have been treated successfully using various techniques of repair. ${ }^{6}$ However, the avascularity of the central or inner one-third of the meniscus results in poor healing at this site. ${ }^{6}$ Tears of the meniscus in this region are generally treated by excision and debridement. Since the introduction of cellular therapy, however, researchers have studied different sources of cells for meniscal repair. ${ }^{7}$ Mauck et $\mathrm{al}^{8}$ evaluated the multipotency of meniscal fibrochondrocytes in vitro. They found that these could be differentiated into chondrocytes, osteocytes and adipocytes, and suggested their use for meniscal repair. Tissue engineering provides a potential means for repair of meniscal tears and possibly meniscal regeneration. ${ }^{9}$ Over the last few years mesenchymal-stem-cell (MSC)based therapies for the regeneration of different tissues have gained acceptance. The importance of MSCs in cell-based therapy has been reported extensively. ${ }^{10-12}$ The use of autologous MSCs also eliminates any potential problem with immunogenicity and rejection. Studies on the pluripotency and plasticity of MSCs show them to be an ideal candidate for possible augmentation of meniscal healing in this central region. ${ }^{13-15}$

We have investigated the use of MSCs to enhance the healing of avascular meniscal tears in a porcine model.

\section{Materials and Methods}

The study proposal was accepted by our institution's hospital animal experimental research ethics committee.

Determination of porcine meniscal vascularity. Under general anaesthesia the femoral artery of a one-year-old Yorkshire pig was isolated. A cannula was inserted into the artery and $50 \mathrm{ml}$ of liquid barium sulphate was injected. The pig was killed and the hind limb excised and refrigerated. The following day the menisci were excised and $\mathrm{x}$-rayed. ${ }^{16}$ The vascular pattern was compared with that of the human meniscus. 
Isolation, culture and ascertaining of MSCs. The ilium of locally bred, one-year-old, Yorkshire pigs was trephined and marrow aspirated from which MSCs were isolated using a validated, published method. ${ }^{17}$ The cells were cultured to $1 \times 10^{6}$ or $2 \times 10^{6}$ during the following two weeks since this number of cells has been found to be the optimum required for the regeneration of joint tissue. ${ }^{18}$ Fibrin glue (Tisseel VH Fibrin Sealant, Baxter Healthcare Corporation, Westlake Village, California) was used as a carrier for the stem cells. ${ }^{19,20}$ In order to characterise the pluripotential nature of the stem cells derived from the bone marrow, in vitro studies were performed to induce the cells into osteogenic, chondrogenic and adipogenic lineages as previously described. ${ }^{19}$ These cell lines were confirmed by histological staining. We have previously demonstrated that our cultured MSCs, when combined with fibrin glue, can survive and differentiate for up to eight weeks after implantation in a rabbit model. ${ }^{21}$

Tripotential differentiation of MSCs. Adipogenic differentiation was induced by culturing MSCs for two weeks in adipogenic medium (Dulbecco's Modified Eagle's Media containing $10 \%$ Fetal Bovine Serum $1 \mu \mathrm{M}$ dexamethasone, $0.5 \mathrm{mM}$ isobutylmethylxantine, $10 \mu \mathrm{g} / \mathrm{ml}$ of insulin and $100 \mu \mathrm{M}$ indometacin) and assessed using an Oil Red O stain (Sigma-Aldrich, St Louis, Missouri). Osteogenic differentiation was induced by culturing the MSCs for two weeks in an osteogenic medium, DMEM containing $10 \%$ FBS, $100 \mathrm{nM}$ dexamethasone, $10 \mathrm{mM}$ glycerophosphate and $50 \mu \mathrm{M}$ ascorbic acid, and examining it for extracellular matrix calcification by Alizarin Red stain (SigmaAldrich). Chondrogenic differentiation was induced using the micromass culture technique. Briefly, $3 \times 10^{5}$ cells were pelleted into a $15 \mathrm{ml}$ tube and cultured in chondrogenic medium of DMEM containing $1 \%$ FBS, $50 \mu \mathrm{g} / \mathrm{ml}$ of ascorbic acid, $10 \mathrm{ng} / \mathrm{ml}$ of transforming growth factor- $\beta 1$, and $6.25 \mu \mathrm{g} / \mathrm{ml}$ of insulin, for three weeks. Chondrogenesis was confirmed by staining with Toluidine Blue (SigmaAldrich). ${ }^{19}$

Animal model. There were two control groups and one experimental group. Each pig underwent surgery on both hind limbs under general anaesthesia and received antibiotic prophylaxis. The knee was exposed through a midline incision of approximately $7 \mathrm{~cm}$. Medial and lateral skin flaps were raised and a medial parapatellar approach was used. Excision of excess fat ensured adequate exposure. The patella was subluxed laterally and the knee hyperflexed to improve exposure. The medial meniscus was then incised sharply in an oblique direction starting from the free margin and extending peripherally for two-thirds of its width. The incision was located at the junction of the anterior one-third and posterior two-thirds. One hind limb underwent the treatment operation while the other had one of the two control operations according to randomisation by computergenerated numbers.

In the control group $1(\mathrm{n}=9)$ a false operation was performed. The animals had no further treatment of the meniscal tear and the knee was closed in layers using a standard suture. In the control group $2(\mathrm{n}=19)$, which served as a true control, the tear was repaired using a nylon monofilament suture (Prolene; Ethicon, Somerville, New Jersey) in a horizontal mattress configuration and an injection of $2 \mathrm{ml}$ of commercially available (Tisseel VH Fibrin Sealant; Baxter Healthcare Corporation) given to the site of the tear. In the experimental group $3(n=28)$, the meniscal tear was repaired with a horizontal mattress suture and an injection of fibrin glue containing at least 1 to $2 \times 10^{6} \mathrm{cul}-$ tured autologous MSCs. Each knee was closed in the standard manner using absorbable Vicryl sutures (Ethicon). The animals were allowed to roam freely within their enclosures post-operatively and were given analgesia and antibiotics according to the protocol.

Macroscopic and microscopic assessment. A pilot study was conducted prior to the full experiment, using an additional set of eight pigs to determine the optimal time of death. The pilot study followed the treatment protocol in which two each were killed at four, six, eight and 12 weeks. Optimal healing was found to occur macroscopically at eight weeks and no further improvement was seen after an additional four weeks. With the results of the pilot study, the timing of death for the remaining 26 pigs (the two killed at eight weeks in the pilot study were included in the total number of the experimental group) was set at eight weeks.

Menisci from the three groups were examined macroscopically and microscopically by an independent investigator (PFC) blinded to the surgery. The macroscopic results were graded according to the system of Henning, Lynch and Clark $^{22}$ whereby healing was compared with the thickness of the cut in the meniscus. Failure was graded as healing $<50 \%$, incomplete healing as $>50 \%$ and complete healing was considered as $>90 \%$. For microscopic assessment, the specimens were fixed with paraffin, cryopreserved and sectioned with a microtome parallel to the meniscal surface. They were stained with haematoxylin and eosin for morphological analysis and Masson's trichrome (Both: Sigma-Aldrich) for the presence of cross-linked collagen, and then examined under light microscopy.

Biomechanical testing. A total of 12 pigs additional to the 28 in group 3, were allocated for biomechanical testing. From these animals six menisci each were harvested which had been treated according to the control group 1, control group 2 and experiment group 3 protocols with an added normal group, where no procedure was undertaken. These pigs were killed at eight weeks. The menisci were trimmed to form a rhomboid shape for ease of testing and the crosssectional area was determined. The ends of the menisci were stitched to commercially available sandpaper to interface with the toothed vice grip of an Instron 5543 universal materials testing machine (Instron Corporation, Norwood, Massachusetts). Tensile testing of the menisci was performed to determine the mechanical properties. Young's modulus of elasticity ${ }^{23}$ was calculated using the cross-sectional area of the specimen and by observing the change in 
length of the specimen in relation to the tensile force applied. The differences in Young's modulus were statistically compared for variance.

Cell labelling and detection. The stem cells isolated and expanded from a further two additional pigs were labelled with commercially available carboxyfluorescein diacetate (Vybrant SFDA SE Cell Tracer, Molecular Probes Inc, Eugene, Oregon). At least $1 \times 10^{6}$ labelled cells were combined with Tisseel fibrin glue and administered to a medial meniscal tear created in the left knee of these two pigs in the same manner as for the main study. The tear was repaired with a Prolene suture (Ethibond). The right knee of these two pigs underwent repair of the medial meniscus with a Prolene suture and fibrin glue to serve as a control. At two weeks the pigs were killed and the menisci were removed. The tissue was sectioned, fixed in acetone and the slides were washed in phosphate-buffered saline. They were examined under confocal microscopy (Olympus FV500 model IX70; Olympus, Tokyo, Japan) using a $488 \mathrm{~nm}$ argon ion laser for evidence of fluorescence.

Statistical analysis. Using the chi-squared test, a sample size calculation concluded that 28 pigs would be required to detect a standard difference of 0.78 between the treatment and control groups with a power of $90 \%$ and a $5 \%$ significance $(\mathrm{p}<0.05)$. The analysis of results was performed using the Mann-Whitney U test and analysis of variance with SPSS version 13.0 software (SPSS Inc., Chicago, Illinois).

\section{Results}

Tripotential differentiation of bone marrow-derived MSC. The tripotential differentiation of expanded porcine MSCs derived from bone-marrow was ascertained by their ability to differentiate into cartilaginous matrix, calciumphosphate mineral and oil-producing chondrocytes, osteoblasts and adipocytes, respectively.

Macroscopic. All the pigs were killed at eight weeks. There was complete failure of healing in the nine knees in the control group 1 (Fig. 1a). There were 12 failures and seven incompletely healed menisci (Fig. 1b) in the control group 2. There was incomplete healing in five menisci and complete healing in 21 in the experimental group 3 (Fig. 1c). Two showed failure of healing. In the healed menisci a faint indentation was still visible on close inspection but at the repair site a very tight gap was all that remained of the former incision. The difference in the results in the experimental group compared with each control groups (Fig. 1d) was statistically significant $(\mathrm{p}<0.001)$.

Histological. In control group 1, failure of healing was obvious. The cut edges of the menisci were lined by a single layer of flattened spindle-like cells with a gap between the two lines of cells (Fig. 2a). In control group 2, failure of healing gave similar findings to those of control group 1 . Incomplete healing had two distinct patterns. There was a central non-healing region with a defect lined by a layer of spindle-like cells and a peripheral area showing healing with collagen and fibrochondrocyte-like and fibroblast-like cells. The residual defect was marked by a line formed by several layers of spindle-like cells (Fig. 2b). In the experimental group 3 the examples with incomplete healing had the same appearance as those in group 2. Complete healing resulted in a healed defect showing increased cellularity with signs of regeneration at the injured site. The repopulated cells resembled fibrochondrocyte-like cells (Figs 2c and $2 \mathrm{~d}$ ) and this re-population with fibrocartilage-like tissue was observed in 21 of the 28 MSC-treated menisci. In some cases the location of the defect was marked by a line formed by several layers of spindle-like cells, but in others this line was not present. At higher magnification a wellorganised matrix was observed in 14 MSC-treated menisci with complete cellularity, fibrochondrocytes being the predominant cells (Fig. 2d). Masson's trichrome staining of the treated tissue sections showed the presence of collagen in the matrix of the repaired area (Fig. 2e).

Biomechanical testing. The mean modulus of elasticity of the non-violated porcine meniscus was $122.2 \mathrm{~N}$ (SD 20). In control group 1 the mean modulus was $0.1 \mathrm{~N}$, in control group 2 it was $3.4 \mathrm{~N}$ and in the experimental group 3 it was $1.5 \mathrm{~N}$. Comparison between the mean modulus of elasticity showed that there was a significant improvement in the experimental group compared with the control groups 1 ( $\mathrm{p}<0.001$ and 2 ( $\mathrm{p}<0.002$; Fig. 3 ).

Results of confocal microscopy. Fluorescence was noted in the MSC-treated menisci. This reflected the presence of carboxyfluorescein diacetate-labelled stem cells in the specimen (Fig. 4). Fluorescence was absent from the control group 2 menisci.

\section{Discussion}

We have shown the ability of MSCs to enhance meniscal repair in the knee in a porcine model. In clinical practice acceleration in the healing time after meniscal repair would be beneficial in allowing an earlier return to an active lifestyle.

There have been attempts to augment the healing process in meniscal tears in the avascular zone by the creation of vascular access channels (trephination), the application of fibrin clots, synovial tissue grafting and the use of growth factors. ${ }^{24-27}$ Although the results have been promising, complete restoration of the native meniscus has not yet been accomplished. However, these methods have some disadvantages since native meniscal tissue can be damaged by trephination and healing is achieved by fibrovascular scarring, rather than by the formation of fibrocartilage.

The transplantation of articular chondrocytes seeded on to devitalised meniscal slices and into a meniscal lesion has resulted in the good integration of the scaffold material into the native meniscus. This has promoted the formation of new cartilage matrix in numerous areas. ${ }^{28}$ These results indicate the potential of a cell-based treatment to repair a tear in the avascular zone of the meniscus. However, the extracellular matrix synthesised by the articular chondrocyte differs from that of the meniscal chondrocyte in both composition and biomechanical properties. Therefore the 


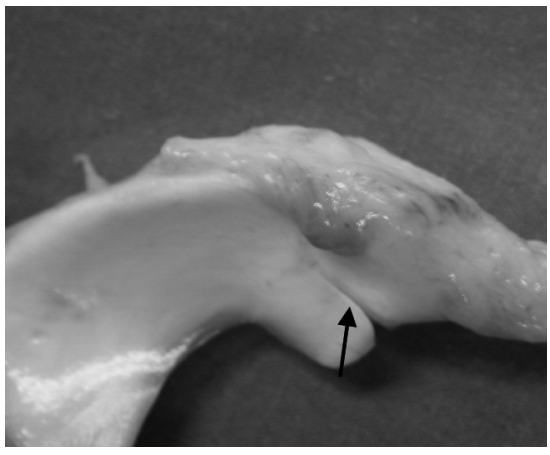

Fig. 1a

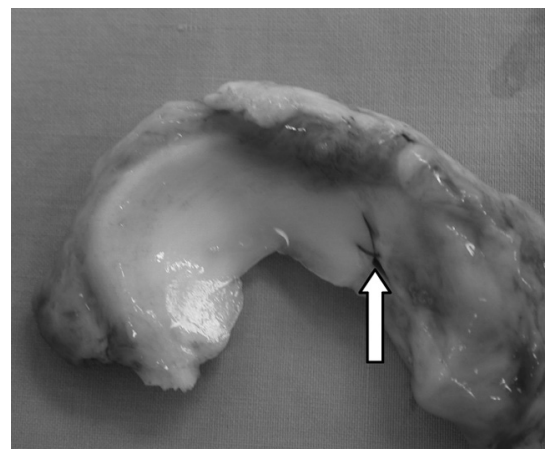

Fig. 1b

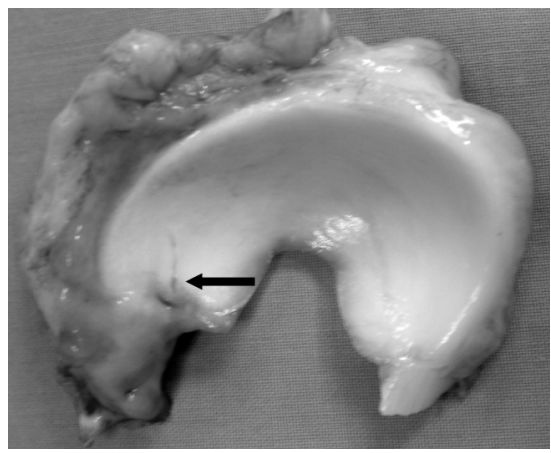

Fig. 1c

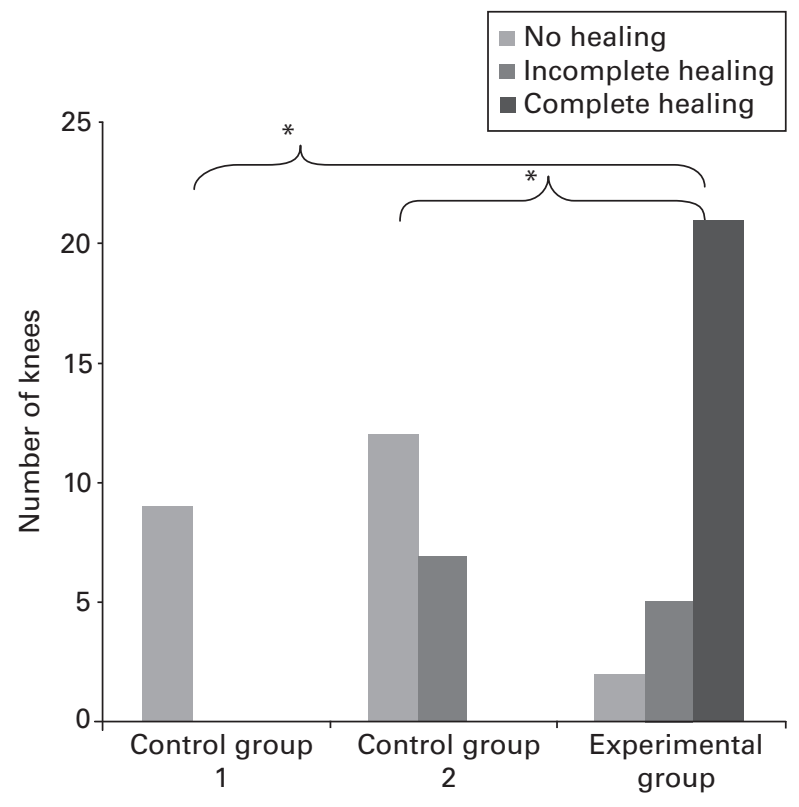

Fig. 1d

Photographs of gross assessment of meniscal healing showing a) failure (small block arrow). b) incomplete healing after repair by suturing and fibrin glue alone (white block arrow). c) complete healing after repair by mesenchymal stem cells, suturing and fibrin glue (black block arrow) and a histogram d) indicating the result of macroscopic grading of the menisci (* significant, Mann-Whitney $U$ test, $p<0.001$ ).

utilisation of a different cell source, such as meniscal fibrochondrocytes may be beneficial. ${ }^{8}$

With their ability to form several tissue types, their expandability and their plasticity MSCs bring great promise, especially in musculoskeletal tissue regeneration. ${ }^{29,30}$ Apart from the ability of MSCs to differentiate into various mesenchymal lineages, the intrinsic secretory activity of MSCs also establishes a regenerative microenvironment at sites of tissue injury or damage. MSCs secrete bioactive factors which inhibit scarring and apoptosis and stimulate angiogenesis and mitosis of tissue-intrinsic stem or progenitor cells. ${ }^{31}$ Autologous MSCs can be obtained from bone marrow without the necessity for an additional arthroscopic procedure to harvest meniscal tissue, thus lowering the risk of further impairment to the normal meniscus.
The ability of MSCs to regenerate meniscal tissue was first explored using an ex vivo organ-culture system ${ }^{14,32}$ in which MSCs derived from rat bone marrow were transplanted into the defects of an explanted meniscus. These underwent in vitro culture to assess their ability to proliferate and to synthesise extracellular matrix. The in vivo repair of a meniscal defect by MSCs was first attempted by Angele et $\mathrm{al}^{33}$ on a critical-size defect in the medial meniscus in a rabbit model. It was found that the repair of the meniscal defect with pre-cultured MSC-scaffold implants integrated with the host tissue and formed meniscus-like fibrocartilaginous tissue. A scaffold comprised of esterified hyaluronan and hydrolysed collagen was used loaded with MSCs, which had been pre-cultured in chondrogenic medium before insertion into the defect. This resulted in significantly better filling of the meniscal defect and 


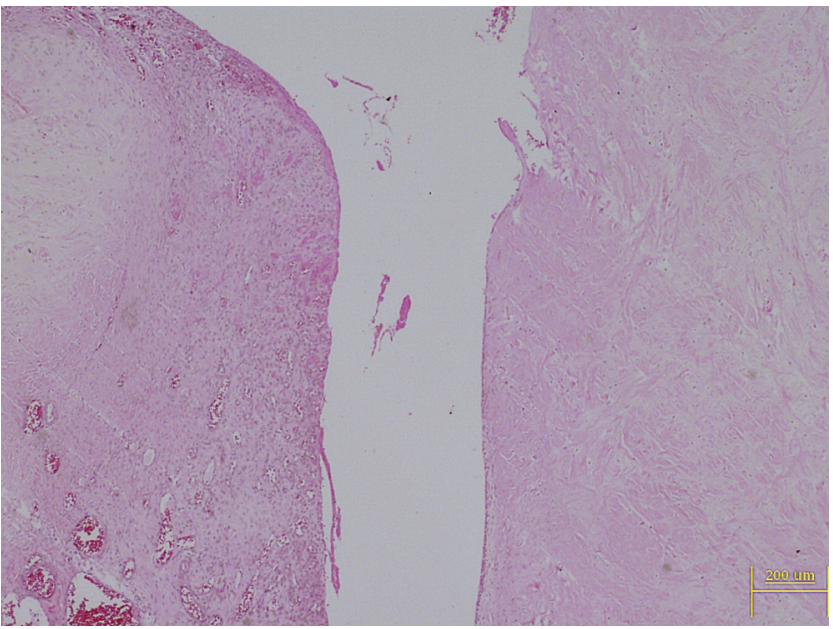

Fig. 2a

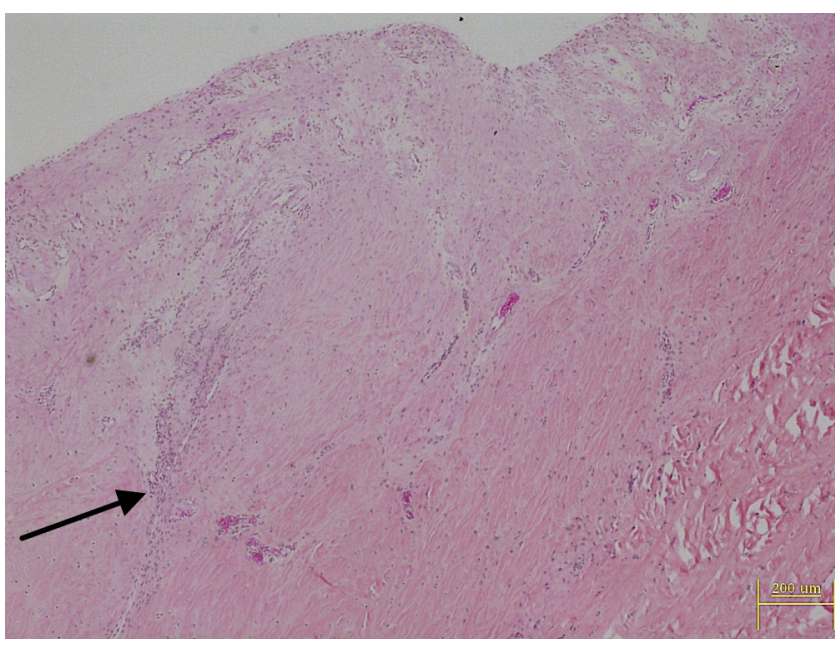

Fig. 2c

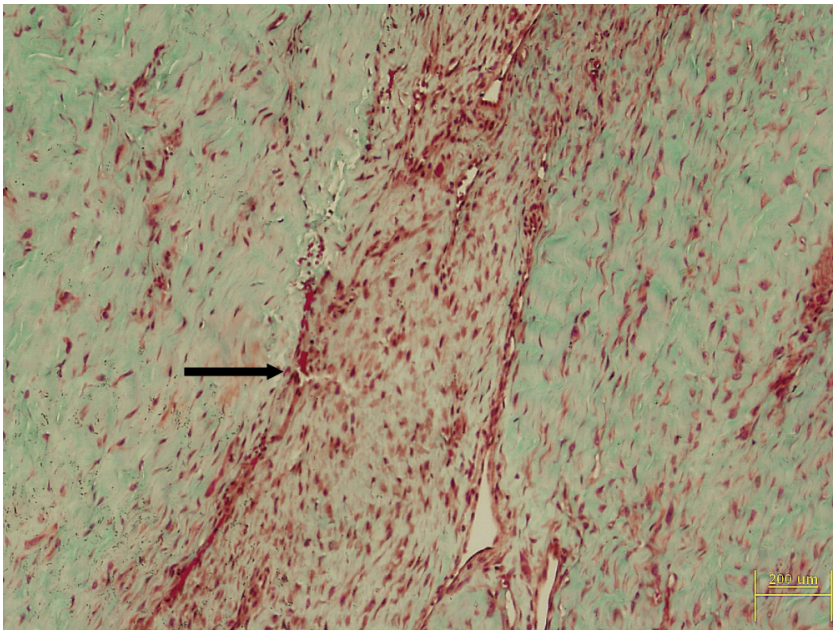

Fig. $2 e$

meniscal regeneration compared with the repair after implantation of cell-free composites.

We have used a large animal model whereas earlier studies have used small mammals ${ }^{14,32,34}$ which are known to

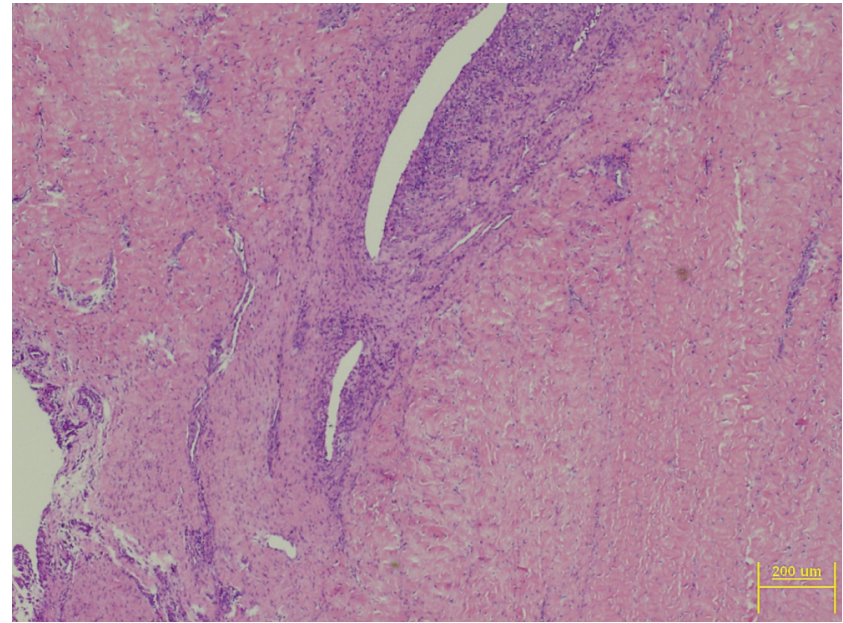

Fig. $2 b$

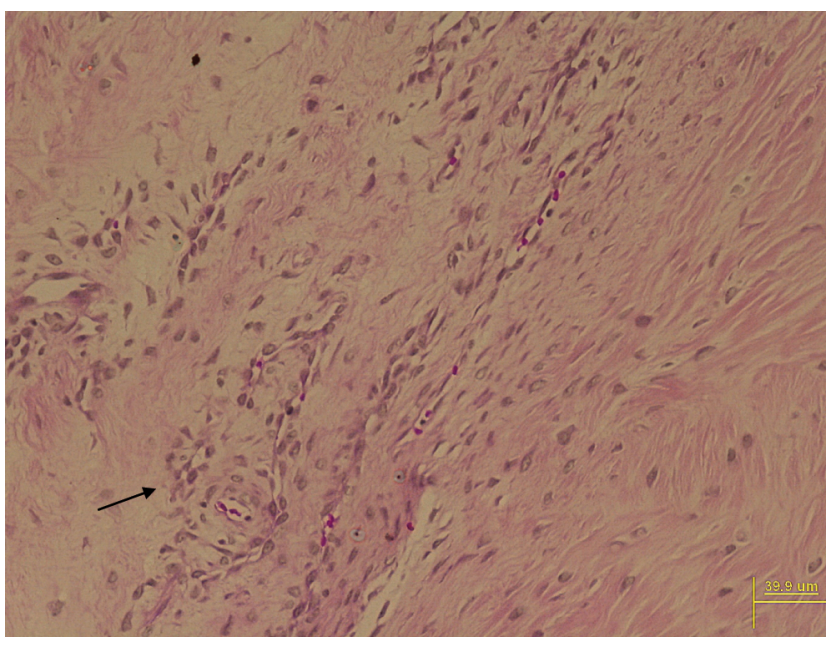

Fig. 2d

Photomicrograph using haematoxylin and eosin staining showing a control group 1 with failure of repair at the meniscal tear $(\times 40)$, b) control group 2 with incomplete healing at the meniscal tear $(\times 40), c)$ treatment group with complete healing and the formation of predominantly fibrocartilage-like tissue (black arrow) at eight weeks $(\times 40), d$ ) treatment group with fibrochondrocyte-like cells $(\times 200)$, and e) formation of matrix at the site of the repair (Masson trichrome staining $\times 100)$.

have an increased healing potential, compared with that of the human. Extrapolation of such results to a human situation may therefore not be appropriate. The pig is more closely comparable with the human in terms of healing 


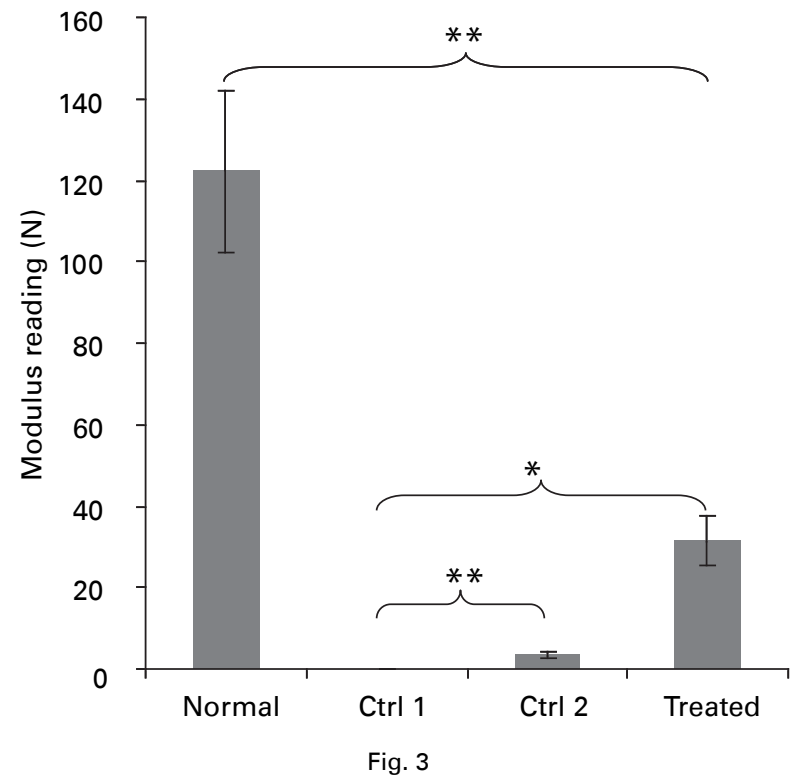

Histogram indicating the modulus of elasticity of each group and the normal porcine meniscus. The results are shown as the mean with the error bars representing the SD $*^{*}$, significance at $p<0.005 ;{ }^{*}$, significance at $\mathrm{p}<0.002$ (analysis of variance)).

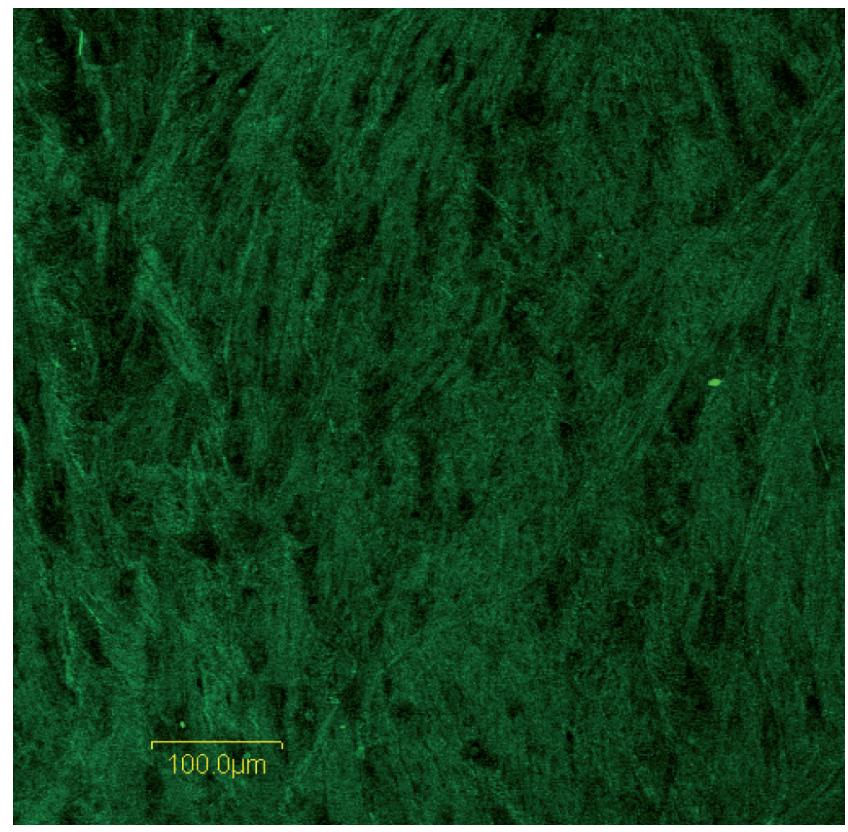

Fig. 4

Confocal microscopy at two weeks showing carboxyfluorescein diacetate-labelled mesenchymal stem cells in the treated meniscus producing fluorescence.

potential, anatomical structure, and is closer in weight. Our results of microangiography of the porcine meniscus showed that the vascular pattern was similar to that of the human meniscus with a central avascular zone. Similar results have been published by Peretti et al. ${ }^{28}$ In contrast to most previous studies which used contained or punchedout lesions ${ }^{32,34}$ we created a meniscal beak tear since this is a common pattern of clinical presentation. Beak tears are considered to be uncontained since they extend to the inner margin of the meniscus. As such, there is poorer healing potential for these tears compared with other patterns of meniscal tear.

We found that the introduction of MSCs into the tears in the avascular zone of the porcine meniscus produced macroscopic and microscopic healing. Normal meniscal tissue demonstrates both fibroblastic and fibrochondrocytic features which vary in different parts of the meniscus. Fibrocartilage-like tissue was observed in the completely healed menisci after MSC treatment. The pattern of cellularity and formation of collagen matrix in those menisci suggests that the regeneration was due to the MSCs. Increased cellularity showed healing in progress and the predominant cells found were fibroblasts and fibrochondrocytes. Well-organised collagen was also found in the MSC-treated menisci. By comparison, in the absence of MSC, failure of healing was common and the only healing which occurred was incomplete.

Macroscopic healing must be accompanied by the reestablishment of the mechanical properties of the meniscus. Biomechanical testing of our healed menisci showed a significant biomechanical improvement in the MSC-treated menisci compared with those in which no MSCs had been applied. However, the improvement only constituted about $25 \%$ of the normal biomechanical properties as expressed by Young's modulus. A study by Newman et al, ${ }^{23}$ has shown the use of mechanical testing for the functionality assessment of the repaired meniscus in the dog. However, the study only included menisci repaired by suture and the repaired meniscus healed with fibrovascular scar tissue after 26 weeks, with little resistance to compressive displacement.

Our study has shown that avascular meniscal tears treated with autologous MSCs in the porcine model can achieve good macroscopic healing with the formation of fibrocartilage-like tissue and improved biomechanical properties. Our time limit of eight weeks could be one limitation of the study, since the determination of the duration for optimal healing was based purely on gross macroscopic evaluation. It is possible that after eight weeks a more mature type of fibrochondrocyte tissue would provide better biomechanical results. It was noted in a clinical study by Henderson et $\mathrm{al}^{35}$ using autologous chondrocytes to manage focal articular defects of the knee, that limited shortterm gain at three months was followed by signs of improvement at 12 and 24 months. This could have been due to the early post-operative inflammatory process impeding the differentiation of the transplanted cells. Therefore, longer follow-up may be appropriate to define the healing pattern and to establish the safety of administered MSCs in vivo. Future studies should examine the long-term effects of in vivo transplanted MSCs to determine the possible better long-term benefit of our 
approach, and exclude any risk of malignancy. Further improvement in the performance of transplanted MSCs may include using a scaffold other than the fibrin glue used in our study, and the provision of a bioactive factor together with the MSC/scaffold implant, may enhance the repair further.

This work was supported by a grant from Biomedical Research Council, Singapore. We thank the Tissue-Engineering Laboratory at the National University of Singapore and Department of Experimental Surgery, Singapore General Hospital for their technical assistance.

No benefits in any form have been received or will be received from a commercial party related directly or indirectly to the subject of this article.

\section{References}

1. Mills PM, Wang Y, Cicuttini FM, et al. Tibio-femoral cartilage defects 3 to 5 years following arthroscopic partial medial meniscectomy. Osteoarthritis Cartilage 2008;16:1526-21.

2. Kettelkamp DB, Jacobs AW. Tibiofemoral contact area: determination and implications. J Bone Joint Surg [Am] 1972;54-A:349-56.

3. Walker PS, Erkman MJ. The role of the meniscus in force transmission across the knee. Clin Orthop 1975;109:184-92.

4. Rangger C, Klestil T, Gloetzer W, Kemmler G, Benedetto KP. Osteoarthritis after arthroscopic partial meniscectomy. Am J Sports Med 1995;23:240-4

5. Weiss CA, Lundberg M, Hamberg P, DeHaven KE, Gillquist J. Non-operative treatment of meniscal tears. J Bone Joint Surg [Am] 1989;71-A:811-22.

6. Heckmann TP, Barber-Westin SD, Noyes FR. Meniscal repair and transplantation: indications, techniques, rehabilitation, and clinical outcome. J Orthop Sports Phys Ther 2006;36:795-814.

7. Angele $\mathbf{P}$, Johnstone $\mathbf{B}$, Kujat $\mathbf{R}$, et al. Stem cell based tissue engineering for meniscus repair. J Biomed Mater Res A 2008;85:445-55.

8. Mauck RL, Martinez-Diaz GJ, Yuan X, Tuan RS. Regional multilineage differentiation potential of meniscal fibrochondrocytes: implications for meniscus repair. Anat $\operatorname{Rec}$ 2007;290:48-58.

9. Buma P, Ramrattan NN, van Tienan TG, Veth RPH. Tissue engineering of the meniscus. Biomaterials 2004;25:1523-32.

10. Solchaga LA, Yoo JU, Lundberg M, et al. Hyaluronan-based polymers in the treat ment of osteochondral defects. J Orthop Res 2000;18:773-80.

11. Ponticiello MS, Schinagl RM, Kadiyala S, Barry FP. Gelatin-based resorbable sponge as a carrier matrix for human mesenchymal stem cells in cartilage regeneration therapy. J Biomed Mater Res 2000;52:246-50.

12. Lee EH, Hui JH. The potential of stem cells in orthopaedic surgery. J Bone Joint Surg [Br] 2006;88-B:841-51.

13. Pittenger MF, Mackay AM, Beck SC, et al. Multilineage potential of adult human mesenchymal stem cells. Science 1999;284:143-7.

14. Yamasaki T, Deie $\mathbf{M}$, Shinomiya $\mathbf{R}$, et al. Meniscal regeneration using tissue engineering with a scaffold derived from a rat meniscus and mesenchymal stromal cells derived from rat bone marrow. J Biomed Mater Res A 2005;75:23-30.

15. Abdel-Hamid M, Hussein MR, Ahmad AF, Elgezawi EM. Enhancement of the repair of meniscal wounds in the red-white zone (middle third) by the injection of bone marrow cells in canine animal model. Int J Exp Path2005:86:117-23.
16. Tan BK, Ng RTH, Tay NS, Tan BS. Tissue microangiography using a simplified barium sulphate cadaver injection technique. Ann Acad Med Singapore 1999:28:152-4.

17. Ouyang HW, Goh JCH, Lee EH. Use of bone marrow stromal cells for tendon graftto-bone healing: histological and immunohistochemical studies in a rabbit model. Am J Sports Med 2004;32:321-7.

18. Agung M, Ochi M, Yanada S, et al. Mobilization of bone marrow-derived mesenchymal stem cells into the injured tissues after intraarticular injection and their contribution to tissue regeneration. Knee Surg Sports Traumatol Arthrosc 2006;14:1307-14.

19. Lee KB, Hui JH, Song IC, Ardany L, Lee EH. Injectable mesenchymal stem cell therapy for large cartilage defects: a porcine model. Stem Cells 2007;25:2964-71.

20. Lim JK, Hui JH, Li L, et al. Enhancement of tendon graft osseointegration using mesenchymal stem cells in a rabbit model of anterior cruciate ligament reconstruction. Arthroscopy 2004;20:899-910.

21. Ouyang HW, Goh JCH, Lee EH. Viability of allogeneic bone marrow stromal cells following local delivery into patella tendon in rabbit model. Cell Transplant 2004;13:649-57.

22. Henning CE, Lynch MA, Clark JR. Vascularity for healing of meniscus repairs. Arthroscopy 1987;3:13-18

23. Newman AP, Anderson DR, Daniels AU, Dales MC. Mechanics of the healed meniscus in a canine model. Am J Sports Med 1989;17:164-75

24. Hashimoto J, Kurosaka M, Yoshiya S, Hirohata K. Meniscal repair using fibrin sealant and endothelial cell growth factor: an experimental study in dogs. Am J Sports Med 1992:20:537-41.

25. Okuda K, Ochi M, Shu N, Uchio Y. Meniscal rasping for repair of meniscal tear in the avascular zone. Arthroscopy 1999;15:281-6.

26. Van Trommel MF, Simonian PT, Potter HG, Wickieqicz TL. Arthroscopic meniscal repair with fibrin clot of complete radial tears of the lateral meniscus in the avascular zone. Arthroscopy 1998:14:360-5.

27. Zhang Z, Arnold JA, Williams T, McCann B. Repairs by trephination and suturing of longitudinal injuries in the avascular area of the meniscus in goats. Am J Sports Med 1995;23:35-41.

28. Peretti GM, Gill TJ, Xu JW, Randolph MA, Zaleske DJ. Cell-based therapy for meniscal repair: a large animal study. Am J Sports Med 2004;32:146-58.

29. Hui HP, Ouyang HW, Hutmacher DW, Goh JCH, Lee EH. Mesenchymal stem cells in musculoskeletal tissue engineering: a review of recent advances in National University of Singapore. Ann Acad Med Singapore 2005;34:206-12.

30. Murphy JM, Fink DJ, Hunziker EB, Barry FP. Stem cell therapy in a caprine model of osteoarthritis. Arthritis Rheum 2003;48:3464-74

31. Caplan Al. Adult mesenchymal stem cells for tissue engineering versus regenerative medicine. J Cell Physiol 2007;213:341-7.

32. Izuta Y, Ochi M, Adachi M, et al. Meniscal repair using bone marrow-derived mesenchymal stem cells: experimental study using green fluorescent protein transgenic rats. Knee 2005;12:217-23.

33. Angele $\mathbf{P}$, Johnstone $\mathbf{B}$, Kujat $\mathbf{R}$, et al. Stem cell based tissue engineering for meniscus repair. J Biomed Mater Res A 2008:85:445-55.

34. Walsh CJ, Goodman D, Caplan AI, Goldberg VM. Meniscus regeneration in a rabbit partial meniscectomy model. Tissue Eng 1999;5:327-37.

35. Henderson I, Francisco R, Oakes B, Cameron J. Autologous chondrocyte implantation for treatment of focal chondral defects of the knee: a clinical, arthroscopic, MRI and histologic evaluation at 2 years. Knee 2005;12:209-16. 